\title{
MedienPädagogik
}

Zeitschrift für Theorie und Praxis der Medienbildung

\section{What if und What will ... be like?}

\section{Spekulative Taktiken der Zukunftsexploration in einer postdigitalen Gegenwart}

\author{
Robert Hausmann ${ }^{1}$ (๑) \\ ${ }^{1}$ Burg Giebichenstein Kunsthochschule Halle
}

\section{Zusammenfassung}

Gegenwärtiges In-der-Welt-Sein scheint vom Zukünftigen, vom Spekulativen, von ungleichzeitigen und permanent vorläufigen Bedingungen bestimmt zu werden. Digitalität hat Subjekte, Räume und Alltagspraktiken durchdrungen. Mitten in diesen postdigitalen Verhältnissen entwerfen nicht nur zeitgenössische oder besser gesagt «zukunftsgenössische» Künstlerinnen und Künstler wie Mary Maggic (Avanessian und Malik 2016, 24), sondern auch TikTokerinnen und TikToker Szenarien des What-if und What-will-...-be-like. Sie erzeugen dabei spekulative Spielräume für (Un-)Möglichkeiten und Projektionen von Zukünften im Hier und Jetzt. Im Beitrag werden diese Modi von Zukünftigkeit in den Blick genommen und danach gefragt, wie diese Taktiken des Erweiterns und handelnden Erprobens von Zukünften neue Impulse für Lernen und Bildungsprozesse eröffnen. 


\title{
What if and What will ... be like? Speculative Tactics of Future Exploration in a Post-Digital Present
}

\begin{abstract}
Present being-in-the-world seems to be determined by the future, by the speculative, by uneven and permanently provisional conditions. Digitality has permeated subjects, spaces, and everyday practices. In the midst of these postdigital conditions, not only contemporary or rather "future-contemporary» artists such as Mary Maggic (Avanessian and Malik 2016, 24), but also TikTokers design scenarios of what-if and what-will-...-be-like. In doing so, they create speculative scopes for (im)possibilities and projections of futures in the here and now. In this article, we will look at these modes of futurity and ask how these tactics of expanding and actively testing futures open up new impulses for learning and educational processes.
\end{abstract}

\section{Postdigitales Festival}

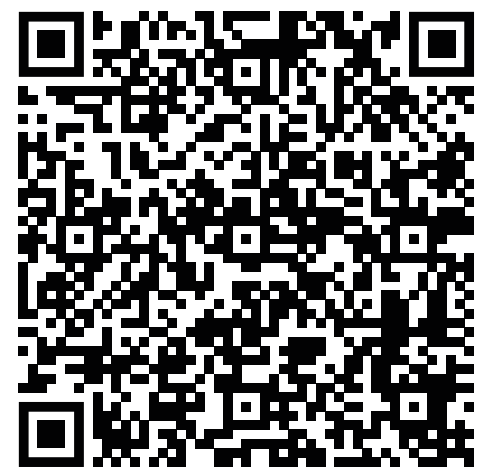

QR-Code 1: TikTok-Video «Class of 2025». https://bit.ly/3wrbDE3.

Das TikTok-Video Class of 2025 von zubeidahkananu (Originalton: Teachers in 2026) zeigt einen spekulativen Umgang mit dem Hier und Jetzt. Gegenwart wird darin auf eine mehr oder weniger ironisierende Weise in eine 
mögliche Zukunft weitererzählt. Eine potenziell künftige Situation einer Lehrperson vor einer Schulklasse wird visualisiert. Dabei werden gegenwärtige Bedingungen mit gespielter Ernsthaftigkeit adaptiert und bestehende Machtkonstellationen - die Idee von Schule, das Morgenritual, der Habitus der Lehrperson, die Frontalität, der tradierte 〈Schulraum〉 - mehr oder weniger unreflektiert reproduziert. Das Video ist Teil eines grösseren Bildkomplexes, in dem Agierende zum Originalton lipsyncen und das Dargestellte im Vorbild-Nachbild-Modus auf ähnliche oder abgewandelte Weise performen. TikTok ist ein weit verzweigtes, dichtes und pausenlos verfügbares «Festivalgelände» (Meier 2021), auf dem sich Akteurinnen und Akteure quer durch Musik, Tanz, Inszenierung, Performance, Schauspiel, Comedy oder Persiflage bewegen und dabei gewohnte Grenzen zwischen den Sparten der Künste aufheben und für überflüssig erklären. Sie erzählen Witze, sie ahmen nach, wiederholen, touren sich gegenseitig in Challenges hoch, zeigen sich Dinge, interagieren in Duetten, sie lipsyncen, tanzen, performen oder spekulieren im Was-wäre-wenn und Wie-wird-es-sein über alternative Realitäten und potenzielle Zukünfte. Das Videoportal ist als mobile App verfügbar, ermöglicht zeit- und ortsungebundenes Liken, Streamen und Posten und bietet verschiedene Möglichkeiten zur Erstellung von maximal 60 Sekunden langen Kurzvideos, die im Loop laufen. Der KI-gesteuerte TikTok-Algorithmus und das Taggen von Videos mit \#foryou sorgen für eine zusätzliche virale Streuung. In der Aufführungspraxis und Videoproduktion verlassen die Akteurinnen und Akteure mitunter herkömmliche Vorstellungen von Qualität. Es geht weniger um die Genauigkeit der Darstellung, um die Perfektion der Nachahmung oder der technischen Bearbeitung, sondern vor allem um authentische Übersetzungen, um alternative, bisweilen trickreiche Bewegungen, leichte, spielerische oder ungewöhnliche Moves, um die unkomplizierte Machbarkeit, eine schnelle Ausführbarkeit und Wiederholbarkeit. Der Wiedererkennungseffekt sowie Anschluss- und Verknüpfungsmöglichkeiten der Handlungen sind zentral. Von Interesse sind auch die unmittelbare Verfügbarkeit und unkomplizierte Modifizierbarkeit digitalen (Roh-)Materials: So können Akteurinnen und Akteure zu bereits verfügbaren Tonspuren oder selbst erstellten Audiosequenzen Videos produzieren und sie parallel bzw. zeitgleich durch GreenScreen-Technik, Videoschnitt, Zeit- und Raumraffungen, Schrift, Filter oder 
Spezialeffekte bearbeiten. Dabei ist auch die Arbeit im Vorbild-NachbildModus von Bedeutung, durch die ganze Bildkomplexe zu ein und derselben Tonspur und Handlung entstehen. Appropriation und Rekombination sind Normalität und verbreitete Methoden der Produktion (vgl. Stalder 2017, 67). Denn Videos auf TikTok sind Teil vernetzter, kollektiver Praktiken: Sie zirkulieren, sie werden rezipiert, Content wird aufgegriffen, nachgeahmt und dabei verändert. Produktion, Postproduktion und Rezeption, d. h. das Machen, Bearbeiten, Anschauen und Teilen sind ineinander verwoben und laufen gleichzeitig ab, wie Benjamin Jörissen, Karoline Schröder und Anna Carnap in ihrer Forschung zu ästhetischen Praktiken einer postdigitalen Jugendkultur aufzeigen (Jörissen, Schröder, und Carnap 2020, 72f.).

Im Folgenden gehe ich zunächst näher auf das zugrunde liegende Verständnis der diesen Beitrag leitenden Begriffe Zukunft, Zukunftsentwürfe, Spekulation und Taktik ein, um diese anschliessend mit medialen Praktiken spekulativer Zukunftsexploration zwischen zeitgenössischen bzw. «zukunftsgenössischen» (Avanessian und Malik 2016, 24) künstlerischen Positionen am Beispiel von Mary Maggic und Videos auf TikTok in Bezug zu setzen. Augenscheinlich wird dabei eine aktuelle Konjunktur des Spekulativen im Modus von Zukünftigkeit unter postdigitalen Bedingungen. An diese vorläufige Beobachtung anknüpfend werden schliesslich Implikationen für eine (kunst- und medien-)pädagogische Praxis, Potenziale des Spekulativen im Kontext von Lernprozessen und Anschlussfragen für eine gegenwärtige kulturelle Bildung abgeleitet.

\section{Zukunft, Zukunftsentwürfe, Zukünftigkeit, Spekulation}

Zukunft ist eine nächste, eine «zukünftige Gegenwart» (Esposito 2016a, 41). Genauer gesagt und den Plural berücksichtigend ist Zukunft ein unabschliessbarer Horizont aller zukünftigen Gegenwarten, die noch kommen werden, der sich gewissermassen mit jeder Gegenwart ständig aufs Neue ausweitet. Zukunft bzw. eine künftige Gegenwart ist also das, was irgendwann tatsächlich Realität sein wird. Zukunftsentwürfe, -szenarien und Vorhersagen wiederum sind gegenwärtige Bilder von Zukunft, «gegenwärtige Zukünfte» (Esposito 2016a, 41). Entwerfen ist überdies eine Praxis, die 
grundsätzlich auf Zukunft, auf ein Noch-nicht ausgerichtet ist. Zukunftsentwürfe arbeiten im Modus von Zukünftigkeit, d. h. sie bewegen sich in einem Zustand des In-der-Zukunft-Seins. Dabei sagen sie mehr über das Jetzt aus als über ein tatsächliches Morgen. Sie sind vom Hier und Jetzt ausgehende projizierte Bilder von Möglichkeiten oder Unmöglichkeiten, die gleichzeitig wieder auf das Jetzt zurückgeworfen werden. Deshalb sind Zukunftsentwürfe «[...] hervorragende Indikatoren für das Verständnis jener Gegenwart, die sie hervorbringt» (Vosskamp 2013, 15). In Spekulationen wiederum wird etwas vermutet und entworfen, was noch nicht ist, aber das Potenzial besitzt, Realität zu werden. Spekulative Entwürfe im What-if und What-will-be-...-like projizieren etwas (Anders-)Mögliches und Zukünftiges ins Hier und Jetzt.

\section{Mögliche Zukünfte}

Zukunft ist noch unerschlossenes Terrain. Sie ist von Offenheit, Unbestimmtheit und Ungewissheit geprägt. Niemand weiss, was tatsächlich passieren wird. Zukünftige Gegenwarten sind, so die Soziologin Elena Esposito, «unbestimmte Bereiche des Möglichen» und Zukunft ist deshalb ein «Zustand mehrfacher Kontingenz». Die Unbekanntheit der Zukunft birgt Risiken, die trotz aller Planbarkeit, aller Routinen und Absehbarkeiten eingegangen werden (vgl. Esposito 2016a, 40f.). Selbst in routinierten Situationen treten immer wieder neu «[...] jene Zehntelsekunden, zuweilen auch Minuten auf, in denen alle wissen, dass niemand weiss, was als Nächstes passiert» (Baecker 2012, 6). Offen ist Zukunft auch, weil niemand mit Gewissheit sagen kann, wie sich andere menschliche Akteurinnen und Akteure sowie nichtmenschliche Agierende und Entitäten in Bezug auf sie verhalten werden (vgl. Esposito 2007, 52).

In einer Welt, in der potenziell alle - menschliche wie nichtmenschliche Agierende - Entscheidungen treffen können, von deren Konsequenzen wiederum andere abhängig sind, die ihrerseits Entscheidungen treffen, potenzieren sich Möglichkeiten, aber auch Ungewissheiten. Zukunft wird in diesen Verstrickungen auch immer zu einem Horizont kollektiv entworfener Unsicherheiten (vgl. Esposito 2007, 52). In allen gegenwärtigen Situationen, die in die Zukunft gerichtet sind, geht es, so Dirk Baecker, darum, 
das Hier und Jetzt «[...] mit jener Unsicherheit anzureichern, die die einen unruhig macht und die anderen auf Ideen bringt» (Baecker 2012, 6). Die jeweilige Gegenwart bietet somit trotz aller (Zukunfts-)Pläne und Routinen Spielräume für Möglichkeiten, die andersmögliche Zukünfte erzeugen können. Das entwirft ein Bild einer nicht nur offenen und undurchsichtigen, sondern auch einer risikobehafteten, unsicheren Zukunft (Esposito 2007, 51).

Zukunft ist kein hermetisch abgeschlossener Zeit-Raum, keine «unerkennbare Leere» (Paglen 2015, 225), die nicht existiert, weil sie noch nicht passiert ist. Sie bietet auch keine utopische Offenheit - was allzu oft mit der Aussage «in der Zukunft ist noch alles möglich» verbunden wird. Zukunft ist von vergangenen Ereignissen und gegenwärtigen Reaktionen menschlicher und nichtmenschlicher Agierender, von Entscheidungen, Handlungen und deren Rückkopplungen abhängig. Sie ist weder ein «neutraler Raum» noch ein «unbegrenzter Möglichkeitsraum», vereinfacht gesagt: Morgen oder übermorgen könnte tatsächlich nur das möglich werden, was gestern, vorhin, gerade eben oder jetzt vorbereitet wurde (Esposito 2016b, 32). Das heisst, vergangene Ereignisse, Entwicklungen und gegenwärtige Bedingungen schreiben gewisse Koordinaten möglicher Archäologien von Zukünften ins Hier und Jetzt ein: Algorithmen, künstliche Intelligenz, technologische Weiterentwicklungen, ständige Updates und Hybridisierungen von Mensch und Technologie, eine mit Stickstoff und Kohlendioxid angereicherte Atmosphäre, gerodete Wälder, schmelzende Polkappen, radioaktive Abfälle, eine fallengelassene Zigarettenkippe, die Sushi-Box aus Plastik oder endokrine Disruptoren etwa in Pflegeprodukten, Kunststoffen, Pestiziden und Düngemitteln ziehen nicht nur eine vermeintlich «toxische Zukunft» (Tsang 2017, 12) nach sich. Sie werden, so der Künstler und Geograf Trevor Paglen, zu nichtmenschlichen, "geschichtlichen Akteuren, die ihre eigenen Zukünfte erzeugen» (Paglen 2015, 224). Mary Maggic $^{1}$ beispielsweise zeigt in ihrer künstlerischen Arbeit und Forschung auf, wie ein Zusammenwirken endokriner Stoffe und menschlicher wie

1 Mary Maggic (Mary Tsang) ist eine nonbinäre Künstlerin und Biologin, die an der Schnittstelle zwischen transfeministischer Kunst, Biotechnologie und Gender Hacking arbeitet und dabei Spekulatives Design als taktisches Tool nutzt. In spekulativen Untersuchungen regt Maggic unentwegt zum biologischen zivilen Ungehorsam an. Siehe auch: https://maggic.ooo. 
nichtmenschlicher Organismen auf molekularer Ebene zu Modifikationen von Körpern, zur Entstehung von bodies in between und neuer bio-queerer Möglichkeiten führt, die herkömmliche Vorstellungen einer binären, heteronormativen natürlichen Ordnung unterwandern und grundsätzlich in Zweifel ziehen (Tsang 2017, 7, 12). Maggic spekuliert deswegen über eine neue Normalität, eine queering landscape, in der sich Körper an wandelnde Bedingungen anpassen, um resilient für eine denkbar toxische Zukunft zu werden: «Adapting, not barricading» sei das Motto (Tsang 2017, 12).

Zum einen schreiben derartige Koordinaten aus der Vergangenheit heraus der Gegenwart gewisse Ausrichtungen potenzieller Zukünfte vor, was Zukunft eben nicht ganz offen macht: Man könnte auch sagen, dass ein Teil der Zukunft sich bereits in der Vergangenheit ereignet hat (vgl. Paglen 2015, 225). Zum anderen werden hier und jetzt mit jeder möglichen Handlung oder Reaktion auf diese Bedingungen wiederum neue Rückkopplungen, Eventualitäten und (anders-)mögliche Zukünfte erzeugt, die in ihrem Zusammenspiel, vom Jetzt-Punkt aus betrachtet, völlig undurchschaubar sind. Im Unterschied zu utopischen oder dystopischen Szenarien, die eher in wünschenswerten oder weniger wünschenswerten Zukünften denken, wird ein grosser Teil tatsächlicher Zukunft von vergangenen wie gegenwärtigen Ereignissen und Handlungen abhängig sein. Es geht also um ein Verhältnis zur Zukunft, das sich, so Donna Haraway, weder einer «gameover-Haltung» noch einer bedingungslosen Technikgläubigkeit ${ }^{2}$ verschreibt, sondern das im wirklichen Sinne des Wortes gegenwärtig ist und Unruhe bewahrt (Haraway 2018, 13) - ein Zukunftsverhältnis also, das sich der Unsicherheit, Undurchschaubarkeit und relativen Offenheit bewusst ist. Das spricht für einen Realismus, der sich der Zukunft spekulativ annähert und das Jetzt aus der Zukunft her betrachtet, um sich der Kontingenz von Gegenwart bewusst zu werden und gegenwärtige Handlungsoptionen zu explorieren. In ihrem spekulativen Verhältnis zur Zukunft machen Künstlerinnen und Künstler wie Mary Maggic hierfür Vorschläge. Sowohl in pädagogischer Hinsicht als auch im Kontext kultureller Bildung - und nicht nur hier - würde es mit einem solchen spekulativen Verhältnis zur Zukunft darum gehen, nicht Held oder Heldin zu spielen, «als wüsste man

2 Beispielsweise verbunden mit der Aussage: «Neue Technologien werden schon alles regeln». 
schon, wie es weitergeht», sondern «[...] Entscheidungen zu treffen, die eine Gegenwart ausloten, die auf die Überraschungen der Zukunft vorbereitet ist» (Baecker 2012, 6).

\section{Spekulative Gegenwart}

Dass es also trotz ihrer Unbekanntheit möglich ist, Zukunft jenseits statistischer Wahrscheinlichkeiten und Prognosen in Form von Entwürfen und Szenarien im Modus des What-if und What-will-...-be-like auf eine spekulative Weise zu explorieren, rückt Spekulationen und Zukünfte unter gegenwärtigen Bedingungen in den Fokus von Lern- und Bildungsprozessen. Nicht nur, aber vor allem seit dem Speculative Turn (Bryant, Srnicek, und Harman 2011) in Philosophie und Kulturwissenschaft gehört die Spekulation zum Vokabular der Gegenwart (Gramlich 2020, 15f.; u. a. Sehgal 2017). Eine Gegenwart der Digitalität potenziert das Spekulative und das Hereinholen von Zukunft ins Hier und Jetzt. Digitalität hat Menschen und Dinge, Räume und Alltagspraktiken durchdrungen. Wir bewegen uns mittendrin, können den weiteren Verlauf nicht abschätzen, weil unzählige menschliche und nichtmenschliche Agierende daran beteiligt sind, die miteinander agieren und aufeinander reagieren. Diese Dynamik versetzt das Hier und Jetzt in einen Zustand permanenter Vorläufigkeit. Folglich wird unaufhaltsam spekuliert. Wir leben in einer spekulativen Gegenwart, die im Sinne Armen Avanessians und Suhail Maliks konstruiert wird «[...] durch die Ungewissheiten der Zukunft und die Abwesenheit der Vergangenheit» (Avanessian und Malik 2016, 22). Digitale Kulturen sind, so Martina Leeker, vom Spekulativen, vom Zukünftigen durchdrungen: Sie nehmen vorweg, greifen vor, beruhen auf Antizipation (Leeker 2020, 162, 169).

\section{Spekulation und Taktik}

In diesem Modus denken und handeln auch jene Akteurinnen und Akteure, deren spekulative Narrative in diesem Beitrag angeteasert werden. Sie gehen auf mehr oder weniger komplexe Weise produktiv mit Unbestimmtheit um, indem sie Verhältnisse zu potenziellen Zukünften spekulativ und taktisch ausloten. Spekulieren wird hier zu einer Praktik der Relationierung, 
des Sich-in-Beziehung-Setzens zu dem, was noch nicht ist. Dieses Nochnicht ist Ausdruck des Unbestimmten und Nicht-Sichtbaren, gegenwärtiger Kontingenz und künftiger Potenzialität. Die Taktik wiederum wird, so Michel de Certeau, «[...] durch das Fehlen von Macht bestimmt, während die Strategie durch eine Macht organisiert wird» (Certeau 1988, 90). Die Taktik tastet sich Schritt für Schritt voran, ohne einen Gesamtüberblick zu haben: «Sie muss mit dem Terrain fertigwerden, das ihr so vorgegeben wird [...]. Sie profitiert von 〈Gelegenheiten〉 [...]» und nutzt Möglichkeiten des Augenblicks (Certeau 1988, 89). Spekulieren wiederum kann in Anlehnung an Reckwitz als ästhetische Praktik der Zukunftsexploration verstanden werden (vgl. Reckwitz 2016, 49). Im Gegensatz zu Prognosen, die Möglichkeitsräume blockieren (vgl. Dörrenbächer 2013, 186), erhalten Spekulationen Zukunft als offenen und kontingenten Horizont, der in gewissem Masse abhängig ist von gegenwärtigen Entscheidungen. Spekulationen bieten Anlässe für ein ludisches Herumlaborieren und Explorieren potenzieller Szenarien. In Bezug zum Spekulativen Design wird deutlich, wie sich ein solches Zusammenspiel von Spekulation und Taktik hinsichtlich der Potenzialität von Zukunft ausrichten kann: «Spekulatives Design», so der Soziologe und Designtheoretiker Benjamin Bratton, «ist eine Zone, in der taktische Abweichungen von den Normen möglich sind und in der deshalb neue Normen getestet werden können» (Bratton 2016, 114). An der Schnittstelle zur kulturellen Bildung stellt sich die Frage, ob auch die ästhetischen Praktiken des Spekulativen in aktueller Kunst und postdigitalen Medienkulturen solche Erprobungsräume für Andersmöglichkeiten und Befragungen gegenwärtiger Verhältnisse eröffnen und somit Testzonen des Performens möglicher Zukünfte erzeugen.

\section{Modi von Zukünftigkeit: Was wäre, wenn mein Toaster mich toasten würde? Oder: Ich, wenn 2075 wieder Clubs öffnen}

Um sich dem gegenwärtigen Phänomen des Spekulativen anzunähern, werden zunächst Vorgehensweisen von Akteurinnen und Akteuren in zwei weiteren TikTok-Videos exploriert. Beide werden anschliessend mit einer komprimierten Analyse der künstlerischen Arbeit Housewives Making 
Drugs von Mary Maggic flankiert, um schliesslich Schnittmengen spekulativer Modi von Zukünftigkeit zwischen Kunst und Medienkultur unter gegenwärtigen postdigitalen Bedingungen zu skizzieren.

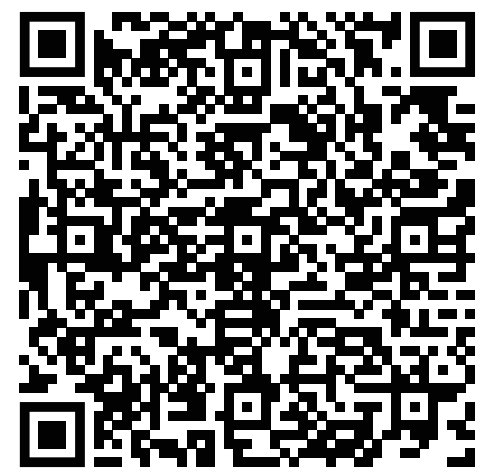

QR-Code 2: TikTok-Video «What if the chair sat on you». https://bit.ly/3zldYT7.

Das Video What if the chair sat on you zeigt ein posthumanes Szenario. Zum Originalton Indy oder unter \#whatifchallenge existieren auf TikTok zahlreiche weitere Szenarien, die ähnlich funktionieren, in denen also nichtmenschliche Akteure auf Menschen in gespiegelter Weise zurückhandeln. Hier werden Szenarien gestaltet und erprobt, die eine nicht-anthropozentrische Perspektive versuchen und sich meist bis hin zu Horror- oder Dark-Fantasy-Erzählungen steigern. Dinge haben darin ihr Eigenleben, sind vermenschlicht und durch Handlungen beseelt. Die Logik, dass Dinge etwas mit Menschen tun, während Menschen etwas mit ihnen tun, wird weder aufgehoben noch umgekehrt, sondern animistisch gesteigert. Das What-if-Szenario wird durch die〈Augen〉 der Dinge gezeigt und durchgespielt. Vorstellungen von menschlicher Autonomie werden dabei grundsätzlich infrage gestellt. In diesen Was-wäre-wennNarrationen wird - in gewisser Weise spielerisch - eine Furcht vor dem Kontrollverlust über Dinge handelnd erprobt (vgl. Dörrenbächer 2016, 12). Anschlussfähig wären hier beispielsweise Theorien der Object-oriented Ontology oder des Agentiellen Realismus. Im Modus des Was-wäre-wenn wird, wie an diesem Beispiel ersichtlich, nicht nur über eine Andersmöglichkeit 
des Daseins, d. h. menschlich-nichtmenschlicher Konstellationen sinniert, sondern zeitgleich eine alternative bzw. spekulative Realität konstruiert und durchgespielt, die auch eine andere Zukunft nach sich ziehen würde.

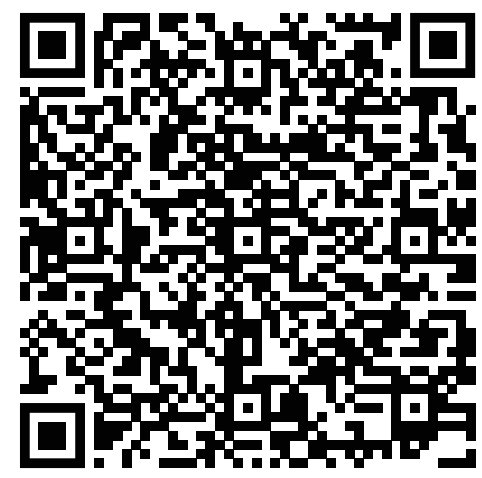

QR-Code 3: TikTok-Video «Parenting in 2050». https://bit.ly/3vrfWoZ.

Das TikTok-Video Parenting in 2050 von jahvonn._ hingegen thematisiert auf ironische, mitunter zynische Weise mögliche Einstellungen und Selbstverständnisse einer Generation, die potenziell in den 2020er-Jahren geboren werden könnte. Spekuliert wird im Modus des What-will-...-be-like, um eine potenziell alltägliche Situation der Kinder- oder besser gesagt 〈Elternerziehung> im Jahr 2040 auf Basis gegenwärtiger gesellschaftlicher Einstellungsgrössen und Entwicklungen zu inszenieren. So wird im ShotGegenshot-Prinzip ein Szenario performt bzw. preenactet, welches sich nicht nur auf der Schneide zwischen Kritik und Gegenkritik einer genderneutralen Sprache und Wirkungen sprachlicher Diskriminierung bewegt, sondern auch die Macht digitaler Kulturen im Kontext von Cancel Culture, Diffamierungen und Shitstorms bearbeitet. 


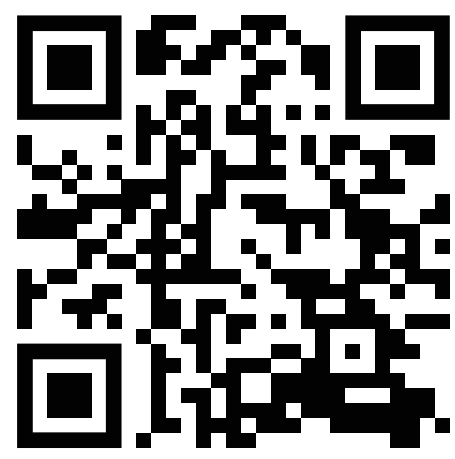

QR-Code 4: «Mary Maggic: Housewives Making Drugs». 2017. YouTube-Miniserie, in Zusammenarbeit mit Jade Phoenix, Jade Renegade und Orgasmic Creative. https://youtu.be/JeyhNquwHKs.

Das spekulative Szenario Housewives Making Drugs des nonbinären Kunstschaffenden Mary Maggic ist eine YouTube-Miniserie aus dem Jahr 2017 und Teil der Reihe Open Source Estrogen. Mary Maggic positioniert in dieser fiktiven und subversiven Kochshow ihre Hauptagierenden, Jade Phoenix und Jade Renegade aka Maria und Maria in die Küche einer eigens für den Dreh angemieteten Airbnb-Wohnung. Anlass ist ein restriktives US-Gesundheitssystem sowie die LGBTQIA+ feindliche Politik der Administration unter Präsident Donald Trump infolge der Abschaffung der Regelung zum Schutz vor Diskriminierung im Gesundheitswesen und damit verbundener Kürzungen in der Gesundheitsversorgung, was eine staatlich unterstützte Hormontherapie für Transmenschen erheblich erschwerte. Die zentrale Frage, die in Housewives Making Drugs gestellt wird, ist deshalb folgende: Was wäre, wenn es möglich sein könnte, Östrogen in der eigenen Küche zu synthetisieren? Mary Maggic stellt dafür ein niedrigschwelliges Rezept bereit, das Biohacking als Do-It-Yourself-Anwendung für zuhause ermöglicht. Mittels einfacher, handelsüblicher Zutaten und Werkzeuge wird aus dem eigenen Urin Östrogen gewonnen. Das aufbereitete Hormon wird anschliessend mit Gin aufgegossen und sich anschliessend als täglicher Shot selbst verabreicht. Die Küche wird zum Heimlabor und, wie Mary Maggic es beschreibt, zum popkulturellen und queeren «Schlachtfeld» für Genderpolitik (Tsang 2017, 39). 
Neben dem Prinzip des Hacks lebt dieses Szenario vom widerständigen Handeln, von Selbstermächtigung, von der Open-Source-Devise Sharing is Caring, aber vor allem vom Spekulieren und Narrativieren aus transfeministischer Perspektive. Maggic nutzt dafür ein bekanntes Format um und verwandelt es in scharfsinniges Trash-TV. Setting, Form und Format rekurrieren nicht nur auf einen historisch-kulturell determinierten Ort des Weiblichen oder auf den Kochshow-Hype der 200oer- und 2010er-Jahre, sondern auch auf die feministische Performance Semiotics of the Kitchen der Künstlerin Martha Rosler aus dem Jahr 1975. Mary Maggic strickt daraus ein Szenario, das sich im Format eines DIY-Tutorials verpackt als taktisch kluges und überraschendes Manöver entgegen heterosexueller Normierungen entpuppt.

Die Agierenden in Housewives Making Drugs stiften nicht nur zum biologischen zivilen Ungehorsam an. Sie hacken ein transphobisches US-Gesundheitssystem, stören binäre Geschlechterkonstrukte und heteronormative Machtstrukturen. Sie erzeugen eine neue Normalität, in der sich Einstellungen rekonfigurieren müssen, weil nach veränderten Wertmassstäben gehandelt wird. Sie spekulieren unter gegenwärtigen Bedingungen mit einer andersmöglichen Zukunft und erproben neue Routinen, die zugleich neue Fragen eröffnen: Was wäre, wenn dieses Was-wäre-wenn-Szenario tatsächlich der Fall werden würde? Housewives Making Drugs wird zur Testzone des Performens einer denkbaren (anderen) Zukunft auf Probe und eben auch dementsprechender Konsequenzen. Maggic löst in diesem Szenario nicht nur institutionalisierte Grenzen zwischen den Sparten der Künste auf, sondern vermischt auf selbstverständliche Weise ästhetische Praktiken aus Kunst, Spekulativem Design und postdigitaler Medienkultur.

In den beschriebenen Beispielen bewegen sich die Akteurinnen und Akteure zwischen Kunst und TikTok in zwei miteinander verstrickten Modi von Zukünftigkeit: im Wie-wird-es-sein und im Was-wäre-wenn. Ihre Herangehensweisen und Aufführungspraktiken weisen Schnittmengen auf: 
1. Sie sind von Inszenierung und Performativität (Fischer-Lichte 2013). bestimmt.

2. Die Akteurinnen und Akteure gehen spekulativ mit gegenwärtigen Bedingungen um. Zum Rohmaterial ihrer Spekulationen werden menschliche Aktivitäten und nichtmenschliche Entitäten, Dinge, Gegebenheiten, Ereignisse, Hormone, Viren, Paradigmen, Machtmechanismen, Krisen oder andere stabile Instabilitäten, mit Latour formuliert: alle im Hier und Jetzt verfügbaren «Dinge von Belang» bzw. «umstrittenen Tatsachen» (Latour 2007, 21f.).

3. Die Agierenden zeigen forschend-explorierende, bisweilen ludische oder hackende Zugänge. Sie bewegen sich in einem temporären Kontinuum zwischen den Zeithorizonten, sozusagen in einer spekulativen Realität, in der sich Gegenwart und potenziell Zukünftiges miteinander vermischen. Sie handeln vor dem Hintergrund einer Art Was-wärewenn-Geisteshaltung. Spekulieren passiert nicht nur in der blossen Vorstellungskraft, die Spekulationen werden in Handlungen und Materie übersetzt. Analog-digitale ästhetische Praktiken hybridisieren sich dabei auf verschiedene Weisen, worin verschiedene Agierende, menschlich wie nichtmenschlich, miteinander verwickelt sind: So wird Zukünftiges bzw. werden Bilder von Zukünften nicht nur imaginiert, sondern zugleich visualisiert und materialisiert und in sinnlich-erfahrbare Wirklichkeiten übersetzt. Die spekulativen Szenerien werden performt und wiederum durch digitale Technologie ${ }^{3}$ und simultane Bearbeitungsmöglichkeiten ${ }^{4}$ transformiert. Zudem nimmt im Fall von TikTok der Algorithmus bzw. die KI als komplexer nichtmenschlicher Akteur unter anderem Einfluss auf Streuung und Sichtbarkeit.

4. In der Überschneidung von Realität und Fiktion, Ernst, Witz und Eigensinn wird in der Erprobung konkreter Szenarien versucht, die Unbestimmtheit von Zukunft durch Bestimmtheit zu ersetzen. Dabei werden neue Unbestimmtheiten produziert.

3 Apps, Software oder in diesem Fall die Tools auf TikTok.

4 Wie z. B. Green-Screen, Filter, Zeitraffer oder Hyperlapse. 
5. Kritik, Reflexion und im Fall von Housewives Making Drugs vor allem widerständige und gegenhegemoniale Narrative verpacken sich in andersmöglichen oder ironisierenden Szenarien, die auf gegenwärtige Bedingungen verweisen. Obwohl mit Zukünftigem spekuliert wird, sagen sie doch einiges mehr über Gegenwart aus.

6. Nicht zuletzt handelt es sich um Formen von Preenactments (vgl. Czirak et al. 2019), die mittendrin in postdigitalen Bedingungen entstehen und auf Basis aktuell verfügbaren Contents arbeiten: Dabei nehmen die Akteurinnen und Akteure auf mehr oder weniger komplexe Weise $\mathrm{Zu}$ künftiges bzw. Etwas-das-so-noch-nicht-ist handelnd vorweg, machen es gegenwärtig und erfahrbar. Das liesse sich als ein «Warm-Up» verstehen (vgl. Marchart 2019, 133), als eine Praxis des Aufwärmens für potenzielle Zukünfte, die wahrscheinlich anders eintreten werden als erwartet, aber deren Gelingens- oder auch Nichtgelingensbedingungen in gewissem Masse abhängig sind von gegenwärtigen Handlungen und Entscheidungen.

\section{Spekulation als Werkzeug und Kritik}

Spekulationen ermöglichen es, potenzielle Zukünfte in der Gegenwart zu imaginieren, zu materialisieren und zu erproben, um darüber gegenwärtige Bedingungen, Diskurse und Verhältnisse aus Perspektive einer potenziellen Zukunft zu denken. Anthony Dunne und Fiona Raby beschreiben die Spekulation deswegen als Tool, als Werkzeug, mithilfe dessen - erwünschte wie unerwünschte - Möglichkeiten von Zukunft entworfen und erfahrbar gemacht werden können, die dabei helfen, Gegenwart neu oder anders zu begreifen und umzudenken:

«[...] [creating] possible futures and using them as tools to better understand the present and to discuss the kind of future people want, and, of course, ones people do not want» (Dunne und Raby 2013, 2f).

Diese Projektionen hinein in eine spekulative Zukunft wirken wiederum auf die Gegenwart zurück und können Räume für Perspektivwechsel oder veränderte Diskurse eröffnen. Auf diese Weise agieren Spekulationen als Critical Friends von Gegenwart. Spekulieren ist dabei eine Praxis der 
Kritik (vgl. Sehgal 2017, 172f). Ein spekulatives Voraussehen in potenzielle Zukünfte und ein gleichzeitiges Zurückgeworfen-Werden auf das Hier und Jetzt ermöglicht kritische Beobachtungen von Gegenwart aus Richtung der Zukunft. Kritik formt sich in Spekulationen folglich nicht aus dem heraus, was war, nicht aus der Vergangenheit und nicht aus sogenannter kritischer Distanz, sondern aus der potenziellen Situation heraus, aus dem Mittendrin-Sein also, in dem, was (anders-)möglich sein oder werden könnte:

«It starts out from what is given in experience, a problem, a situation that forces one to think, then tries to actively change the terms of this problem or situation, in order to change the course of its consequences: What if this problem was answered in this way? What would this lead to?» (Sehgal 2017, 177).

Die gesteigerte Präsenz des Spekulativen in postdigitalen Kulturen zeigt aber auch, das Gegenwart «[...] nicht mehr primär aus der Vergangenheit abgleitet werden [kann], [...] sondern [...] von der Zukunft gestaltet [wird]» (Avanessian und Malik 2016, 12). Die Spekulation ist demnach nicht nur eine Methode kritischen Denkens, mit der sich nach Melanie Sehgal eine «neue Form der Kritik» etabliert (Sehgal 2017, 173; vgl. Leeker 2020, 162), sondern auch eine Methode zur Generierung von Erkenntnissen, die sich in einer spekulativen Haltung zur Wirklichkeit zeigt (vgl. Meyer 2017, 113).

Zukunftsentwürfe aktueller Künstlerinnen und Künstler wie Maggics Housewives Making Drugs sind Spekulationen, die ein Zukunftsverständnis erkennen lassen, das auf Emergenz beruht. Ein emergentes Verhältnis zur Zukunft ist eines, das nicht auf den Eintritt einer bestimmten Zukunft abzielt, sondern Suchbewegungen zulässt und dabei mit «[...] Offenheiten und Ungewissheiten bei der Erzeugung von Zukunftswissen» arbeitet (Schubert 2013, 21). Im Gegensatz zu prognostischen und normativen Zukunftsverhältnissen können die ins real life übersetzten spekulativen Entwürfe und Untersuchungen aktueller Künstlerinnen und Künstler - wie es Hans-Jörg Rheinberger im Kontext wissenschaftlicher Aktivitäten feststellt - im übertragenen Sinn als «Generatoren von Überraschungen» wirken, ohne dass ihre Vermutungen sich bewahrheiten müssten (Rheinberger 1992, 71). 
Bisweilen sind ihre Spekulationen deshalb gegenhegemoniale Entwürfe, Gegenreaktionen auf herrschende Narrative oder aber Weitererzählungen eines Systems, das gegen die Wand läuft (vgl. Gramlich 2020, 23). Als «Medium der Kritik» (Leeker 2020, 162) eingesetzt stimulieren Spekulationen nämlich Dekonstruktionen und Destabilisierungen von Machtverhältnissen oder die Bergung und Sichtbarmachung von Perspektiven, die bisher (noch) nicht erzählt wurden. In solchen spekulativen Gegenzukünften geht es im Gegensatz zu normativen gesellschaftlichen Zukunftsprognosen um die Wiederaneignung von Zukunftsnarrativen, beispielsweise aus eben nicht-weissen und nicht-heterosexuellen Perspektiven im Sinne postkolonialer Kritik.

Spekulationen in postdigitalen Kulturen sind jedoch nicht per se als kritisch im Sinne eines Problematisierens und Hinterfragens gegenwärtiger Verhältnisse zu bezeichnen: So besitzen zukunftsexplorierende TikTok-Videos, die etwa in Narrativen wie \#futuregirlfriend, \#futureboyfriend, \#futuregoals oder \#futurelife agieren, ihre inhaltlichen wie ästhetischen Grenzen, indem sie Zukunft lediglich in bestehenden binären Kategorien weiterdenken und dabei gegenwärtige soziale Konstitutionen, Ungleichgewichte, Klischees und Machtverhältnisse reproduzieren.

\section{Möglichkeitsspiele im Noch-nicht}

Was lässt sich daraus nun für (kunst- und medien-)pädagogische Praxis, für Lernen, Vermittlung und für eine gegenwärtige ästhetische Bildung ableiten?

Spekulative Untersuchungen und Zukunftsentwürfe erweitern den Zukunftshorizont der Gegenwart um Möglichkeiten. "In Summa», bemerkt Thomas Düllo,

«haben die Was-wäre-wenn-Narrationen eine Erkenntnisfunktion für die Gegenwart und ihre weitere Entfaltung, weniger als Prognostik, vielmehr in ihrer Orientierungsfunktion und Eröffnung eines Denkraums des Möglichen an der Grenze zwischen wahrscheinlicher Unwahrscheinlichkeit und unwahrscheinlicher Wahrscheinlichkeit» (Düllo 2013, 32). 
Für Martin Seel bieten Entwürfe von Zukünften deswegen Potenziale, «[...] innerhalb des Wirklichen den Sinn für das Mögliche zu schärfen [...]» (Seel 2001, S. 747). Auch Hans Blumenberg verortete in dieser «Vergegenwärtigung des Nicht-Anwesenden» Momente, die etwas mit Lern- und Bildungsprozessen zu tun haben: Denn die «Vorwegnahme des Möglichen» von morgen sei auch ein «Angebot von Wahlhandlungen» für heute, die ein «Mehr-Wahrnehmen-Können» von Gegenwart ermöglicht (Blumenberg 2007, 26f.). In dieser Praxis des Vorwegnehmens, bei dem Zukunftsentwürfe Gegenwart zugleich mit Kontingenz anreichern, sieht Blumenberg den Anstoss für ästhetische Erfahrungen (Arnold 2018, 4; Blumenberg 2007, 26). Wenn über Zukunftsentwürfe also Feedbacks für und (Neu-)Erfahrungen von Gegenwart möglich werden und sie das Potenzial besitzen, aus Richtung spekulativ entworfener Zukünfte gegenwärtiges Handeln zu einem gewissen Grad neu zu justieren, können sie zu Instrumenten einer «[... Anwartschaft auf neue Gegenwärtigkeit, neue Anschauung [...]» werden (Blumenberg 2007, 27).

Im Entwurf wie auch in der Erprobung spekulativer Narrative geht es folglich darum, rezeptiv wie produktiv Zukunft bzw. Zukünfte als Erfahrung(-en) zu ermöglichen und dabei gleichzeitig das Hier und Jetzt als Differenz wahrzunehmen. In Entwürfen werden Zukünfte präfiguriert, ein Noch-nicht wird im Vorhinein gedacht, imaginiert, visualisiert und materialisiert. Wer entwirft, bewegt sich im Modus des Spekulativen: Was wäre, wenn... Indem potenzielle Zukünfte dabei vorweggenommen oder antizipiert werden, gleich ob sie so eintreten werden oder es - sehr wahrscheinlich eher - ganz anders kommt als erwartet, ereignet sich dabei unweigerlich eine Bewusstwerdung und (Neu-)Wahrnehmung des Hier und Jetzt. Diese Vergegenwärtigung oder «Aktivierung von Gegenwart» ist für Lernprozesse unentbehrlich (Sabisch 2003, 425).

Spekulieren - verstanden als Praktik, die Kontingenzerfahrungen ermöglicht -, kann Prozesse des Lernens initiieren, in denen Lernen mit Bezug auf Meyer-Drawe als «Umlernen» im Sinne eines Erschliessens von Möglichkeiten zu verstehen ist (Meyer-Drawe 1984, 206; vgl. Westphal 2014). Lernen ist demnach als ein Verwickelt-Sein mit Welt zu begreifen, das immer mit dem Risiko verbunden ist, «[...] die Sache sowie unsere Beziehung zum anderen umstrukturieren zu müssen» (Meyer-Drawe 2012, 214). 
In einem aktualisierten Verständnis liesse sich Meyer-Drawes Umlernen mit Nora Sternfelds Verlernen ${ }^{5}$ weiterdenken. Lernen versteht Sternfeld als kritisch-reflexive Praxis des Anzweifelns bestehender Welt- und Selbstverhältnisse, als

«[...] manchmal mühsamen und schmerzhaften, manchmal aufregend-lustvollen Prozess der Überschreitung und des Abarbeitens der antrainierten Sicherheiten, die die Machtverhältnisse tradieren» (Sternfeld 2014, 19).

Verlernen im Sinne spekulativen Andershandelns kann demnach zu einer Art Übung werden, «[...] um langsam und Schritt für Schritt, mit den angelernten Praxen und Gewohnheiten der machtvollen Unterscheidung, die sich in Habitus, Körper und Handlungen eingeschrieben haben, zu brechen» (Sternfeld 2014, 19). Das impliziert auch ein Verständnis vom Lernen, bei dem in Bezug auf Schule historisch gewachsene Rollenverständnisse, Hierarchien, Machtkonstellationen und die damit verbundene Binarität von Lernenden und Lehrenden nicht nur zur Disposition gestellt, sondern grundsätzlich umgeübt, umgelernt werden: Denn die Zukunft ist - und zwar für alle beteiligten Akteurinnen und Akteure gleichermassen - unbekannt.

In Bezug auf Lern- und Bildungsprozesse im Kontext von Pädagogik und Schule würde das bedeuten, Lernen und Vermittlung vom Zukünftigen her zu denken und gemeinsam laborative Settings zu erzeugen, in denen Spekulationen eine Konkretion erfahren. ${ }^{6}$ Das spricht dafür, spekulative Narrative nicht nur zu imaginieren und zu entwerfen, sondern als What-if-Szenarien in Form von spekulativen Untersuchungen, Prototypen und Preenactments in die unmittelbare Wirklichkeit zu übersetzen, zu enacten, physisch durchzuspielen, zu performen und zu testen (vgl. Leeker 2020, 175). Imaginäres, Unsichtbares, bisher Noch-Nicht-Erzähltes und Nichtwahrnehmbares wird dabei in Formen der Sichtbarkeit überführt

5 Sternfeld in Bezug auf Gayatri Spivaks Konzept des Unlearning (Spivak 1990, 42).

6 Das heisst nicht, Vergangenheit zu negieren oder unter den Tisch fallen zu lassen: Wer sich im Modus des Spekulativen bzw. im Zustand von Zukünftigkeit bewegt, setzt sich - das scheint unvermeidlich - mit dem Ist und dem Geworden-Sein auseinander (vgl. Sternfeld 2014, 15). 
und erprobt. Solche tatsächlichen Live-Erprobungen oder Enactments im Modus des What-if verknüpfen, so Martina Leeker, «[...] Reflexion und Spekulation als Basis für konkretes Um-Denken [in der Gegenwart] und die Konstruktion von Alternativen» (Leeker 2018, 25). Es gehe folglich darum, Zukunftsnarrative als Spekulationen einzuführen und zu erproben, die neue bzw. ungewohnte oder ungewöhnliche Perspektiven möglich werden lassen statt normativen Pfaden zu folgen und prognostische oder stilisierte Zukunftsvorstellungen zu verdoppeln. Im Modus einer solchen spekulativen Zukünftigkeit können gegenwärtige Bedingungen zur Disposition gestellt und Gegenwart ebenso in ihrer potenziellen Andersmöglichkeit wahrgenommen werden. Beim Spekulieren agieren Lernende auf diese Weise im Modus möglichen (Anders-)Seins und (Anders-)Werdens. Sie arbeiten im und mit dem Vorläufigen, werden Teilhabende an kritischen Ausund Erhandlungsprozessen, in denen sich Zukunftsentwürfe spekulativ entspinnen, entwickeln und modellieren.

\section{Bildungsmomente und Anschlussfragen}

Spekulationen mit Alternativen und Zukünften wirken wissen- und erkenntnisgenerierend. Spekulatives Voraus- und Andershandeln kann nicht nur Lernprozesse im Sinne einer Exploration unbekannter (potenziell zukünftiger und andersmöglicher) Terrains anstossen, sondern auch Bildungsmomente stimulieren im Sinne eines aktiv gestalteten, produktiven Umgangs mit Gegenwart und den Unbestimmtheiten von Zukunft. Im daraus resultierenden Anders- und Voraushandeln werden zugleich Reflexion und Kritik von Gegenwart möglich. Das führt zu Anschlussfragen: Inwiefern erzeugen jene kollektiven, spekulativen Taktiken auf TikTok immer wieder neue, sich potenzierende Produktionen von Sinn, aber auch Reproduktionen gegenwärtiger Verhältnisse, hegemonialer Machtkonstellationen und Zukunftsklischees? Wenn kulturelle Bildung zur Teilhabe an einer Zukunft befähigt, von der wir jetzt noch nicht wissen, wie sie aussehen wird, einer Zukunft, die aber sicherlich von gegenwärtigen Aktionen und Reaktionen menschlicher wie nichtmenschlicher Akteure abhängen wird, können dann performative Erprobungen potenzieller Zukünfte im What-if und What-will-...-be-like eine Art Warm-up dafür sein? 
Welche Gelegenheiten bietet das Spekulative für gegenwärtige Pädagogik noch? Können tatsächlich auch Bildungsinstitutionen wie Schule und insbesondere kunstpädagogische Prozesse zu Explorationsräumen für Spekulationen und folglich realexperimentelle Testzonen des Performens von Zukünftigkeit werden? Wenn Zukunft von Offenheit, Unbestimmtheit und Ungewissheit geprägt ist, dann erzeugt jeder Moment sie handelnd zu erschliessen, sie zu testen, zu pre-enacten auch neue Fragen, Unbestimmtheiten und Ungewissheiten: Wer oder - etwa im Fall von KI - was spekuliert? Wem gehört Zukunft? Wessen Zukünfte werden aus welcher Perspektive erzählt und wessen nicht? Welche Zukünfte sind gefährdet? Und was, wenn es tatsächlich möglich wäre, Hormonshots in der eigenen Küche zu synthetisieren?

\section{Literatur}

Arnold, Florian. 2018. «Ästhetik als Prävention. Anthropologische Spekulationen im Ausgang von Hans Blumenberg - Medusa -». In Das ist Ästhetik! OnlineAkten zum X. Kongress der Deutschen Gesellschaft für Ästhetik, herausgegeben von Juliane Rebentisch, 1-12. Offenbach am Main. http://www.dgae.de/kongresse/das-ist-aesthetik/.

Avanessian, Armen, und Suhail Malik. 2016. «Der Zeitkomplex». In Der Zeitkomplex. Postcontemporary, herausgegeben von Armen Avanessian und Suhail Malik, 7-36. Berlin: Merve.

Baecker, Dirk. 2012. «Unbekannte Zukunft». changeX, 14. Dezember 2012. https:// catjects.files.wordpress.com/2019/10/unbekannte_zukunft.pdf.

Blumenberg, Hans. 2007. Theorie der Unbegrifflichkeit. Herausgegeben von Anselm Haverkamp. Frankfurt am Main: Suhrkamp.

Bratton, Benjamin H. 2016. «Zum Spekulativen Design». In Der Zeitkomplex. Postcontemporary, herausgegeben von Armen Avanessian und Suhail Malik, 95119. Berlin: Merve.

Bryant, Levi, Nick Srnicek, und Graham Harman, Hrsg. 2011. The Speculative Turn. Continental Materialism and Realism. Melbourne: Re.press.

Certeau, Michel de. 1988. Kunst des Handelns. Berlin: Merve.

Czirak, Adam, Sophie Nikoleit, Friederike Oberkrome, Verena Straub, Robert Walter-Jochum, und Michael Wetzels, Hrsg. 2019. Performance zwischen den Zeiten. Reenactments und Preenactments in Kunst und Wissenschaft. Bielefeld: Transcript Verlag. https://doi.org/10.14361/9783839446027. 
Dörrenbächer, Judith. 2013. «Irritierende Realitäten oder das Potenzial der Utopie. Ästhetische Taktiken zwischen Kunst und Design». In What's Next. Kunst nach der Krise. Ein Reader, herausgegeben von Johannes M. Hedinger und Torsten Meyer, 185-89. Berlin: Kadmos.

Dörrenbächer, Judith. 2016. «Design zwischen Anthropomorphismus und Animismus». In Beseelte Dinge. Design aus Perspektive des Animismus, herausgegeben von Judith Dörrenbächer und Kerstin Plüm, 71-95. Bielefeld: Transcript Verlag. https://doi.org/10.1515/9783839435588-001.

Düllo, Thomas. 2013. «Als-ob im Modus von What if? Der Denkraum des Wahrscheinlich-Unwahrscheinlichen in Science Fiction und anderswo». Ästhetik \& Kommunikation 44 (162/163): 30-41.

Dunne, Anthony, und Fiona Raby. 2013. Speculative Everything. Design, Fiction and Social Dreaming. Cambridge (Massachusetts): MIT Press.

Esposito, Elena. 2007. Die Fiktion der wahrscheinlichen Realität. Frankfurt am Main: Suhrkamp.

Esposito, Elena. 2016a. «Die Konstruktion von Unberechenbarkeit». In Der Zeitkomplex. Postcontemporary, herausgegeben von Armen Avanessian und Suhail Malik, 37-42. Spekulationen. Berlin: Merve.

Esposito, Elena. 2016b. «Realität der Zukunft und künftige Realität». In Die Gegenwart der Zukunft, herausgegeben von Susanne Witzgall und Kerstin Stakemeier, 29-35. Zürich-Berlin: Diaphanes.

Fischer-Lichte, Erika. 2013. «Performing the Future». In Performing the Future. Die Zukunft der Performativitätsforschung, herausgegeben von Erika Fischer-Lichte und Kristiane Hasselmann, 11-23. München: Wilhelm Fink.

Gramlich. 2020. «Feministisches Spekulieren. Einigen Pfaden folgen». In Feministisches Spekulieren. Genealogien, Narrationen, Zeitlichkeiten, herausgegeben von Marie-Luise Angerer und Naomie Gramlich, 9-29. Berlin: Kadmos.

Haraway, Donna J. 2018. Unruhig bleiben. Die Verwandtschaft der Arten im Chthuluzän. Frankfurt am Main: Campus.

Jörissen, Benjamin, Karoline Schröder, und Anna Carnap. 2020. «Postdigitale Jugendkultur: Kernergebnisse einer qualitativen Studie zur digitalen Transformation ästhetischer und künstlerischer Praktiken». In Kulturelle Bildung. Theoretische Perspektiven, methodologische Herausforderungen, empirische Befunde, herausgegeben von Susanne Timm, Jana Costa, Claudia Kühn, und Annette Scheunpflug, 61-78. Münster: Waxmann Verlag. https://doi. org/10.31244/9783830991502.

Latour, Bruno. 2007. Elend der Kritik. Vom Krieg um Fakten zu Dingen von Belang. Zürich-Berlin: Diaphanes.

Leeker, Martina. 2018. (Ästhetische) Vermittlung 2.0. Von Kunst-/Vermittlung und Kritik in digitalen Kulturen. Herausgegeben von Andrea Sabisch, Torsten Meyer, Heinrich Lüber, und Eva Sturm. Kunstpädagogische Positionen 40. Köln. http://kunst.uni-koeln.de/_kpp_daten/pdf/KPP4O_Leeker.pdf. 
Leeker, Martina. 2020. «Speculate-as-speculate-can. Bedingungen von Spekulation als Kritik in digitalen Kulturen». In Feministisches Spekulieren. Genealogien, Narrationen, Zeitlichkeiten, herausgegeben von Marie-Luise Angerer und Naomie Gramlich, 162-77. Berlin: Kadmos.

Marchart, Oliver. 2019. «Zeitschleifen: Politisch-theoretische Überlegungen zu Preenactments und realen Utopien». In Performance zwischen den Zeiten. Reenactments und Preenactments in Kunst und Wissenschaft, herausgegeben von Adam Czirak, Sophie Nikoleit, Friederike Oberkrome, Verena Straub, Robert Walter-Jochum, und Michael Wetzels, 129-39. Bielefeld: Transcript Verlag. https://doi.org/10.14361/9783839446027-009.

Meier, Philipp. 2021. Das Phänomen Tiktok: ein Gespräch mit Philipp Meier - als Chat Interviewt von Dirk von Gehlen. Online. https://www.dirkvongehlen.de/ netz/das-phaenomen-tiktok-ein-gespraech-mit-philipp-meier-als-chat/.

Meyer, Torsten. 2017. «Zeitgenossenschaft». In 1-13 kunstpädagogische Begriffe, herausgegeben von Sara Burkhardt und Marie Newid, 106-19. Kunstpädagogische Knotenpunkte 02. Halle (Saale): Hochschulverlag Burg Giebichenstein Kunsthochschule Halle.

Meyer-Drawe, Käte. 1984. «Lernen als Umlernen: zur Negativität des Lernprozesses». In Lernen und seine Horizonte: phänomenologische Konzeptionen menschlichen Lernens, didaktische Konsequenzen, herausgegeben von Käte Meyer-Drawe und Wilfried Lippitz, 19-45. Frankfurt am Main: Scriptor.

Meyer-Drawe, Käte. 2012. Diskurse des Lernens. 2. Aufl. München: Wilhelm Fink.

Paglen, Trevor. 2015. «Geographien der Zeit (Die letzten Bilder)». In Realismus | Materialismus / Kunst, herausgegeben von Armen Avanessian, Christoph Cox, Jenny Jaskey, und Suhail Malik, 216-26. Spekulationen. Berlin: Merve.

Reckwitz, Andreas. 2016. «Zukunftspraktiken - Die Zeitlichkeit des Sozialen und die Krise der modernen Rationalisierung der Zukunft». In Die Ungewissheit der Zukunft. Kontingenz in der Geschichte, herausgegeben von Frank Becker, Benjamin Scheller, und Ute Schneider, 31-54. Frankfurt am Main: Campus.

Rheinberger, Hans-Jörg. 1992. Experiment. Differenz. Schrift. Zur Geschichte epistemischer Dinge. Marburg/Lahn: Basilisken-Presse.

Sabisch, Andrea. 2003. «Entwerfen〉 oder 〈Die Welt aus Sicht einer Fliege»». In Kunstdidaktisches Handeln, herausgegeben von Klaus-Peter Busse, 1:414-27. Studien zur Kunstdidaktik. Dortmund: Dortmunder Schriften zur Kunst.

Schubert, Cornelius. 2013. «Die Laboratorisierung gesellschaftlicher Zukünfte. Zum Verhältnis von Labor, Feld und numerischen Prognosen sozialer Dynamiken». Navigationen - Zeitschrift für Medien- und Kulturwissenschaften 13 (2): 151-61. https://doi.org/10.25969/mediarep/1250.

Seel, Martin. 2001. «Drei Regeln für Utopisten». Merkur-Sonderheft 5 (Zukunft denken. Nach den Utopien): 747-55. 
Sehgal, Melanie. 2017. «Speculation». In Symptoms of the Planetary Condition: A Critical Vocabulary, herausgegeben von Mercedes Bunz, Birgit Mara Kaiser, und Kathrin Thiele, 173-78. Lüneburg: Meson Press.

Spivak, Gayatri Chakravorty. 1990. «Strategy, Identity, Writing». In The Post-Colonial Critic, herausgegeben von Sarah Harasym, 35-49. New York/London: Routledge.

Stalder, Felix. 2017. Kultur der Digitalität. 3. Aufl. Berlin: Suhrkamp.

Sternfeld, Nora. 2014. Verlernen vermitteln. Kunstpädagogische Positionen 30. Hamburg. http://kunst.uni-koeln.de/_kpp_daten/pdf/KPP3O_Sternfeld.pdf.

Tsang, Mary. 2017. Open Source Estrogen. From biomolecules to biopolitics... Hormones with institutional biopower! Cambridge (Massachusetts): Massachusetts Institute of Technology. http://hdl.handle.net/1721.1/112560.

Vosskamp, Wilhelm. 2013. «Möglichkeitsdenken. Utopie und Dystopie in der Gegenwart. Einleitung». In Möglichkeitsdenken. Utopie und Dystopie in der Gegenwart, herausgegeben von Wilhelm Vosskamp, Günter Blamberger, und Martin Roussel, 13-30. München: Wilhelm Fink.

Westphal, Kristin. 2014. «Phänomenologie als Forschungsstil und seine Bedeutung für die kulturelle und ästhetische Bildung». KULTURELLE BILDUNG ONLINE. https://doi.org/10.25529/92552.121. 